\title{
Monitoring the coordination of aluminium during microporous oxide crystallisation by in situ soft $X$-ray absorption spectroscopy $\dagger$
}

\author{
Andrew M. Beale, ${ }^{a}$ Ad M. J. van der Eerden, ${ }^{a}$ Didier Grandjean, ${ }^{a}$ Andrei V. Petukhov, ${ }^{b}$ Andy D. Smith ${ }^{c}$ and \\ Bert M. Weckhuysen*a
}

Received (in Cambridge, UK) 14th July 2006, Accepted 14th August 2006

First published as an Advance Article on the web 6th September 2006

DOI: $10.1039 / \mathrm{b} 610080 \mathrm{e}$

An in situ cell, which is capable of obtaining time resolved soft X-ray data $(200 \mathrm{eV}<E<3000 \mathrm{eV})$ under hydrothermal conditions has been developed and used to study the selfassembly processes occurring during microporous aluminophosphate crystallization.

Many of the useful physical properties associated with zeotypes, such as ion-exchange capacity, molecular sieving and shape selectivity, originate from the presence of nanometre-sized channels and/or cages within their structure. Although they occur within the Earth's crust, a large demand on their usage has triggered major research efforts on the preparation of synthetic analogues of these minerals, the alteration of their properties by manipulation of their framework and the synthesis of new topologies altogether. ${ }^{1}$ However, the self-assembly steps by which such important materials form are still not well understood and thus the synthesis of new topologies is still something of a hit and miss process. ${ }^{2-4}$ Understanding the critical stages in microporous materials crystallisation is then clearly the first step towards realising the possibility of rational microporous materials design for specific purposes e.g. tailoring a material's properties for a specific catalytic reaction.

Time-resolved in situ experiments using a variety of analytical techniques have been performed in an attempt to obtain such information. ${ }^{2-4}$ The majority of these studies have concentrated on using scattering techniques to follow these processes since they are sensitive to structural ordering in the system. However, since selfassembly processes involve the ordering of atomic or molecular species, spectroscopic techniques can also yield important insight since they are more sensitive. For example, it has been possible to follow the behaviour of a small amount of a hetero-atom (an example being transition metal ions, which can be considered as a 'probe' species) to obtain information on these self-assembly processes. ${ }^{5-7}$ However, such information can be considered at best indirect and is only possible to observe with hetero-atoms, which give a strong spectroscopic signature and which do not influence

${ }^{a}$ Inorganic Chemistry and Catalysis group, Department of Chemistry, Utrecht University, Sorbonnelaan 16, 3508 TC, Utrecht, The

Netherlands.E-mail:b.m.weckhuysen@chem.uu.nl; Fax: +31302531027; Tel: +31 302537400

'van't Hoff Laboratory for Physical and Colloid Chemistry, Department of Chemistry, Utrecht University, Padualaan 8, 3584 CH, Utrecht, The Netherlands

${ }^{c}$ CCLRC Daresbury Laboratory, Keckwick Lane, Daresbury,

Warrington, UK WA4 4AD

$\uparrow$ Electronic supplementary information (ESI) available: Further analysis of XAFS and corresponding SAXS/WAXS data. See DOI: 10.1039/ b610080e the phase formation processes unduly. It is far more useful therefore to follow the crystallisation behaviour of the major structural elements, although this is more difficult to achieve since their spectroscopic signatures are often comparatively weak or less detailed than the corresponding transition metal ion-substituted systems. Perhaps, the most relevant technique to obtain such molecular insight into the assembly processes under hydrothermal conditions is NMR, since it is particularly sensitive to changes in the local structure of the zeolite/zeotype atomic species. Indeed, it has been successfully employed to obtain detailed information regarding the formation of microporous systems. ${ }^{8-10}$ However, the interpretation of NMR data is not always straightforward due to quadrupolar effects or line broadening as a result of interactions with paramagnetic species, such as $\mathrm{Co}^{2+} .{ }^{11}$ In contrast, X-ray absorption spectroscopy is not limited by such problems and is in principle capable of providing information on both the coordination and oxidation state of the absorber.

Here, we describe a newly developed in situ cell, which can be used to follow the synthesis/crystallisation process of materials under hydrothermal conditions via time-resolved in situ Soft X-ray Absorption Fine Structure (SOXAFS) measurements. In these experiments we have used this set-up to record, for the first time and in real-time, Al K-edge SOXAFS spectra of a crystallization process. This was done on Station 3.4, of the SRS Daresbury Laboratory (UK), which was equipped with a $\mathrm{YB}_{66}$ double crystal scanning monochromator. The synchrotron operated at $2 \mathrm{GeV}$ with a typical current of $c a .170 \mathrm{~mA}$. The energy calibration of the monochromator was performed using a Al metal foil. This new set-up was based on the original vacuum vessel, and replaceable sealed in situ cell previously used to obtain soft X-ray data under catalytic conditions, which has been adapated with a hydrothermal reaction 'vessel' (a diagram of the set-up is given in Fig. 1). ${ }^{12,13}$ The hydrothermal reaction chamber comprised a Teflon liner (hollowed to a depth of $2 \mathrm{~mm}$ ), which was mounted on the heating stage (as shown) on the SOXAFS cell. A $10 \mu \mathrm{m}$ graphite $\mathrm{O}$ ring was mounted on top of the Teflon, which acts as a spacer between the Teflon and Kapton. In this way it was ensured that the hydrothermal environment was maintained but that the Kapton was thin enough $(25 \mu \mathrm{m})$ to allow the X-rays to enter and exit. The chamber was mounted at $45^{\circ}$ to the incident beam and the resultant fluorescence signal was detected using a small cylindrical gas proportional counter (GPC) mounted close to the Kapton window at an angle of $90^{\circ}$ from the incident beam and $45^{\circ}$ to the sample. Before the Kapton was mounted in place, the precursor gel prepared some 30 min earlier, $\$$ was filled up to a maximum of ca. $60 \%$ of the total volume $\left(c a .2 \mathrm{~mm}^{3}\right)$. To ensure that the 


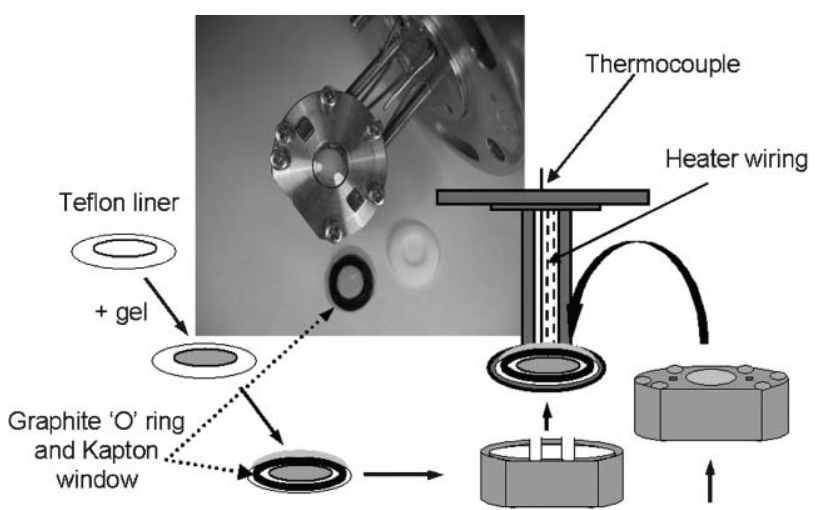

Stainless steel hydrothermal casing

Fig. 1 Schematic of the in situ SOXAFS hydrothermal reactor cell. The schematic illustrates the parts used and steps taken during the charging of the cell with reactant and assembly procedure to produce a suitable hydrothermal reaction environment.

autogeneous pressure necessary for crystallisation was maintained, the heating stage and Kapton window part were subsequently housed within a clamped stainless steel casing. The cell was then heated stepwise via a wound heating element controlled by a thermoregulator at a rate of 3 degrees per min up to $180{ }^{\circ} \mathrm{C}$ and remained at this temperature for up to 50 min under autogeneous pressure and static conditions. The experimental data were obtained in fluorescence yield mode with an energy resolution of $0.3 \mathrm{eV}$ per step around the edge and were normalized to a point $14 \mathrm{eV}$ above the edge. In order to collect data within a reasonable time period, measurements focussed on the XANES region (1558 $1600 \mathrm{eV}$ ), which for $\mathrm{Al}^{3+}$, contains distinct features which allow for facile identification of the different coordination states. ${ }^{14,15} \mathrm{~A}$ counting time of $1.5 \mathrm{~s}$ per point was employed for collecting dynamic XANES data, which resulted in a total scan time of $330 \mathrm{~s}$ per scan (inclusing monochromator deadtime). However, XAFS data to $1850 \mathrm{eV}$ were recorded on the crystallised sample.

In Fig. 2 we show a series of stacked XANES spectra collected during the in situ temperature treatment of a CoAPO synthesis gel.

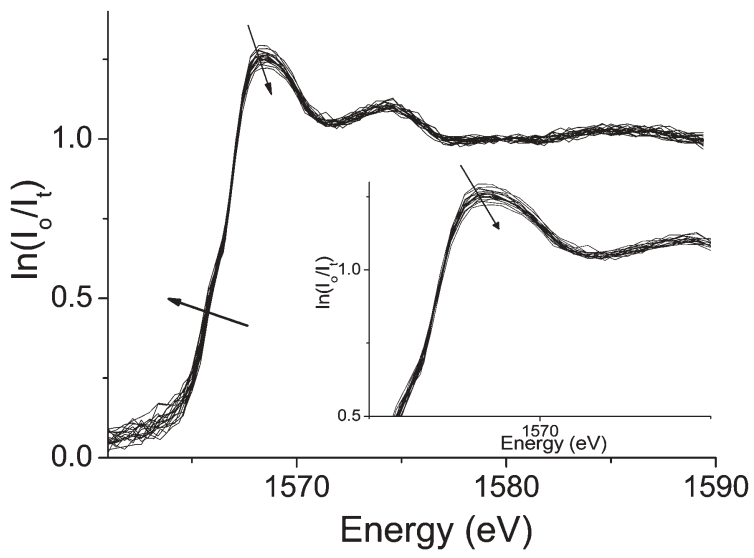

Fig. 2 Normalised in situ $\mathrm{Al} \mathrm{K}$-edge NEXAFS data (and inset figure for the same data which highlights further the changes) in the recorded data during the formation of CoAPO-5. The arrows pointing downwards highlight the decrease in the rising absorption edge intensity as more tetrahedral $\mathrm{Al}^{3+}$ is formed whilst the arrow pointing right-to-left highlight the growth of a feature around $1565 \mathrm{eV}$.
Interestingly, it appeared that $30 \mathrm{~min}$ after preparing the gels and even before heating had commenced, the $\mathrm{Al}^{3+}$ coordination state had changed (as determined by an edge shift from $c a .1568 .1 \mathrm{eV}$ to ca. $1566.9 \mathrm{eV}$ as well as a decrease in the rising absorption edge intensity) from a distorted octahedral coordination typical of the starting pseudoboehmite phase to that more typical of tetrahedral $\mathrm{Al}^{3+}$ (see ESI Fig. 1 for XANES spectra of samples containing $\mathrm{Al}^{3+}$ in both 6- and 4-fold coordination $\dagger$ ). This observation was consistent with previously reported data where dissolution of basic pseudoboehmite occurs readily in phosphoric acid resulting in the formation of an amorphous aluminophosphate precursor gel. ${ }^{7,16}$ However, during the subsequent hydrothermal reaction to form the crystalline AFI type material, a further decrease in the rising absorption edge intensity was observed, which appears consistent with the conversion of more of the remaining octahedral $\mathrm{Al}^{3+}$ centres to tetrahedral coordination. In addition for the CoAPO-5 sample, a much clearer shift in the edge position occurred and a feature at $c a .1565 \mathrm{eV}$ was observed to form and grow. A similar feature has previously been reported by van Bokhoven et al. during steam treatment of zeolite $\beta,{ }^{14}$ which was attributed to the presence of three coordinate (trigonal) $\mathrm{Al}^{3+}$. However, this feature was only observed after treatment at high temperatures and therefore appears unlikely to occur in a hydrothermal environment. A more likely explanation is that $\mathrm{Co}^{2+}$ substitution causes the template to locate closer to the framework (than for pure AlPO-5) or water could interact, which might either induce a distortion of the nearest aluminium site(s) or else an increase in the coordination number resulting in a five-fold coordinated site(s) (most probably of the type trigonal bipyramidal). ${ }^{11,17}$

The significance of the changes in the XANES are better illustrated in Fig. 3, where we show a plot of the rising absorption edge intensity with time for $\mathrm{AlPO}_{4}-5$ and CoAPO- 5 crystallization, plotted alongside the extent of crystallisation/aggregation points as determined in a separate SAXS/WAXS study (see ESI Fig. $3 \dagger$ ). Quantification of the XANES data was performed by comparing the intensity of an octahedral $\left(\mathrm{Al}\left(\mathrm{NO}_{3}\right)_{3} \cdot 9 \mathrm{H}_{2} \mathrm{O}\right)$ and tetrahedral


Fig. 3 Estimation of $\%$ octahedral $\mathrm{Al}^{3+}$ from the rising absorption edge intensity from the in situ $\mathrm{Al} \mathrm{K}$-edge NEXAFS (open squares) and change in the average aggregate/crystallite size (black circles) determined from the SAXS/WAXS data for $\mathrm{AlPO}_{4}-5$ (left) and CoAPO-5 (right). For the CoAPO-5 sample details of the coordination state of $\mathrm{Co}^{2+}$ has also been added (black squares). Above a temperature of $170{ }^{\circ} \mathrm{C}$ (NEXAFS) and $180{ }^{\circ} \mathrm{C}$ (SAXS/WAXS) the $X$-axes correspond to time the samples were maintained at these temperatures. 
(dehydrated zeolite $\mathrm{A}$ ) $\mathrm{Al}^{3+}$ reference compound. The amount of octahedral $\mathrm{Al}^{3+}$ was then obtained by linear combination of the intensities of both coordination contributions. Interestingly, we observe that the overall change to tetrahedral $\mathrm{Al}^{3+}$ during the formation of the final crystalline AFI phases was smaller for the $\mathrm{AlPO}_{4}-5$ sample than for CoAPO-5. For the $\mathrm{AlPO}_{4}-5$ gel it appeared that little changes were observed during the early stages of the aggregation process, but that immediately before and during crystallisation the rising absorption edge intensity declined to a larger extent. A similar behaviour has recently been observed for $\mathrm{Co}^{2+}$ when monitoring the Co K-edge during CoAPO-5 crystallization (for clarity we have included these data in Fig. 3). ${ }^{7}$ However, for the CoAPO-5 material it appeared that a comparatively larger proportion of octahedral $\mathrm{Al}^{3+}$ remained after the initial mixing of the gel, but that the gradual aggregation process and crystallisation was able to force, in a continuous manner, most of the remaining 6-fold coordinated $\mathrm{Al}^{3+}$ to adopt the tetrahedral arrangement necessary for framework formation (Fig. 3). Interestingly, this temperature-dependent behavior is not anymore in line with the data obtained for $\mathrm{Co}^{2+}$, suggesting that $\mathrm{Co}^{2+}$ and $\mathrm{Al}^{3+}$ behaved differently in the aggregation process of CoAPO-5 materials. This lead us to conclude that $\mathrm{Co}^{2+}$ cannot be used as a reliable spectroscopic probe to monitor the crystallization behavior of CoAPO-5 materials. In terms of a plausible crystallization mechanism this could be interpreted that the major transformation from octahedral to tetrahedral $\mathrm{Co}^{2+}$ coincides with CoAPO-5 crystallization, whereas $\mathrm{Al}^{3+}$ is really changing during the initial aggregation with phosphorus. One could argue that this could be related to different crystallization precursors. All this appears therefore to reflect a more 'disruptive' influence by the cobalt species on the gelation process since there is clearly a difficulty in incorporating octahedral cobalt species into the early formed AlPO units. This may lead to an indirect stabilisation of terminal aluminium groups (perhaps as part of 1D-like species or building units) $)^{7,18}$ that would be unable to condense further to form larger aggregates until heating begins.

From analysis of the EXAFS data it was possible to assign quantitative values to the coordination numbers and bond distances. Inspection of these data revealed that the final average coordination numbers and bond distances corroborate the observations seen in the XANES data. In particular the final average $\mathrm{Al}-\mathrm{O}$ distance of $c a .1 .70 \AA$ and coordination number close to 4 for both samples was consistent with the predominance of tetrahedral $\mathrm{Al}^{3+}$ species. ${ }^{19,20}$

In summary, we have developed a new set-up to record SOXAFS (200 eV $<E<3000 \mathrm{eV}$ ) data under hydrothermal crystallization conditions, which has been used to monitor the aggregation and crystallisation processes leading to the formation of microporous solids. This study was able to determine differences in the $\mathrm{Al}^{3+}$ coordination environment between the CoAPO-5 and pure $\mathrm{AlPO}_{4}-5$ gels and it was found that distorted tetrahedral or 5-fold coordinated $\mathrm{Al}^{3+}$ was present in CoAPO-5. Secondly, the method allowed us to estimate the amount of octahedral and tetrahedral $\mathrm{Al}^{3+}$ during the formation processes. The different amounts of octahedral $\mathrm{Al}^{3+}$ suggest that we cannot use $\mathrm{Co}^{2+}$ necessarily as a spectroscopic probe for $\mathrm{Al}^{3+}$ since it seemed to affect the overall formation behavior. $\mathrm{Co}^{2+}$ seemed to affect the aggregates and in doing so retarded aggregation and crystallization. Furthermore, we rationalize that the differences in amount of octahedral $\mathrm{Al}^{3+}$ was due to changes in the nature of the aggregates. It is anticipated that this new technique and related information will help scientists in further developing plausible crystallization mechanisms for porous oxides since it is more generally applicable to any system formed under hydrothermal conditions containing soft elements, such as $\mathrm{Al}^{3+}$.

We acknowledge Kees Baldé, Florian Meneau and the staff members at the DUBBLE beamline for assistance in performing the SOXAFS and SAXS/WAXS measurements. CCLRC is acknowledged for access to station 3.4. Financial support from NWO-CW (VICI project) and beamtime from NWO and EU (translational access contract no. 45041) are kindly acknowledged.

\section{Notes and references}

\$ The following chemicals were used: Cobalt acetate tetrahydrate $\mathrm{C}_{4} \mathrm{H}_{6} \mathrm{CoO}_{4} \cdot 4 \mathrm{H}_{2} \mathrm{O}$ (Acros Organics $99 \%$ ), orthophosphoric acid $\mathrm{H}_{3} \mathrm{PO}_{4}$ (Acros Organics 85 wt.\% in water), triethylamine (TEA) (Acros Organics $99 \%$ ), pseudoboehmite alumina CATAPAL B $73.6 \mathrm{wt} \% \mathrm{Al}_{2} \mathrm{O}_{3}$ (Sasol North America Inc.). The resultant opaque precursor gels corresponding to the stoichiometry $\mathrm{Co}_{x} \mathrm{Al}_{1-x} \mathrm{PO}_{4}$ with $x=0.02$ (cobalt content $\sim 1 \mathrm{wt} \%$ ) were used in the SAXS/WAXS experiments and were prepared by methods described elsewhere. $^{\text {? }}$

1 C. Baerlocher, W. H. Meier and D. H. Olson, Atlas of Zeolite Frameworks 5th Edition, Elsevier, Amsterdam, 2001.

2 C. S. Cundy and P. A. Cox, Microporous Mesoporous Mater., 2005, 82, 1.

3 T. Wakihara and T. Okubo, Chem. Lett., 2005, 34, 276.

4 T. M. Davis, T. O. Drews, H. Ramanan, C. He, J. S. Dong, H. Schnablegger, M. A. Katsoulakis, E. Kokkoli, A. V. McCormick, R. L. Penn and M. Tsapatsis, Nat. Mater., 2006, 5, 400.

5 G. Sankar, J. M. Thomas, F. Rey and G. N. Greaves, J. Chem. Soc., Chem. Commun., 1995, 2549.

6 B. M. Weckhuysen, D. Baetens and R. A. Schoonheydt, Angew. Chem., Int. Ed., 2000, 39, 3419.

7 D. Grandjean, A. M. Beale, A. V. Petukhov and B. M. Weckhuysen, J. Am. Chem. Soc., 2005, 127, 14454.

8 Ø. B. Vistad, D. E. Akporiaye, F. Taulelle and K. P. Lillerud, Chem. Mater., 2003, 15, 1639.

9 C. J. Y. Houssin, C. E. A. Kirschhock, P. Magusin, B. L. Mojet, P. J. Grobet, P. A. Jacobs, J. A. Martens and R. A. van Santen, Phys. Chem. Chem. Phys., 2003, 5, 3518.

10 F. Taulelle, M. Pruski, J. P. Amoureux, D. Lang, A. Bailly, C. Huguenard, M. Haouas, C. Gerardin, T. Loiseau and G. Ferey, J. Am. Chem. Soc., 1999, 121, 12148.

11 D. B. Akoleka and R. F. Howe, J. Chem. Soc., Faraday Trans., 1997, 93, 3263 .

12 A. M. J. van der Eerden, J. A. van Bokhoven, A. D. Smith and D. C. Koningsberger, Rev. Sci. Instrum., 2000, 71, 3260.

13 J. A. van Bokhoven, A. M. J. van der Eerden, A. D. Smith and D. C. Koningsberger, J. Synchrotron Radiat., 1999, 6, 201.

14 J. A. van Bokhoven, A. M. J. van der Eerden and D. C. Koningsberger, J. Am. Chem. Soc., 2003, 125, 7435.

15 J. A. van Bokhoven, H. Sambe, D. E. Ramaker and D. C. Koningsberger, J. Phys. Chem. B, 1999, 103, 7557.

16 R. F. Mortlock, A. T. Bell and C. J. Radke, J. Phys. Chem., 1993, 97, 775.

17 Z. Wu, A. Marcelli, G. Cibin, A. Mottana and G. Della Ventura, J. Phys.: Condens. Matter, 2003, 15, 7139.

18 G. Ferey, J. Solid State Chem., 2000, 152, 37.

19 J. A. van Bokhoven, J. Roelofs, K. P. de Jong and D. C. Koningsberger, Chem.-Eur. J., 2001, 7, 1258.

20 I. J. Drake, Y. Zhang, M. K. Gilles, C. N. Teris Liu, P. Nachimuthu, R. C. C. Perera, H. Wakita and A. T. Bell, J. Phys. Chem. B, 2006, 110, 11665 . 